(Fig. 1). This is in striking opposition with most magnetization reversal models, which consider that in such ultrasmall structures, inhomogeneous excitations are forbidden and that magnetization reversal is only due to homogeneous fluctuations.

These experiments mark a great step towards future ultrahigh-density datastorage technologies. Not only do they demonstrate the control of spin waves down to atomic length scales, but they also give insight to possible read and write processes using spin waves to assist magnetization switching. In the future, the ability to create spin waves in atomic wires should efficiently be used not only for standing waves but also for itinerant spin-waves. This should open avenues for propagating information and transfer the concepts of magnonics ${ }^{12}$ to the atomic scale. Logical operations at the atomic scale, as recently demonstrated ${ }^{13}$, should also benefit from the use of spin waves, opening new perspectives in computing with atoms.

\author{
Stanislas Rohart is at the Laboratoire de \\ Physique des Solides, Université Paris-Sud \\ and CNRS, Bâtiment 510, 91405 Orsay cedex, \\ France. Guillemin Rodary is at Laboratoire \\ de Photonique et de Nanostructures, CNRS, \\ Route de Nozay, 91460 Marcoussis, France. \\ e-mail: stanislas.rohart@u-psud.fr
}

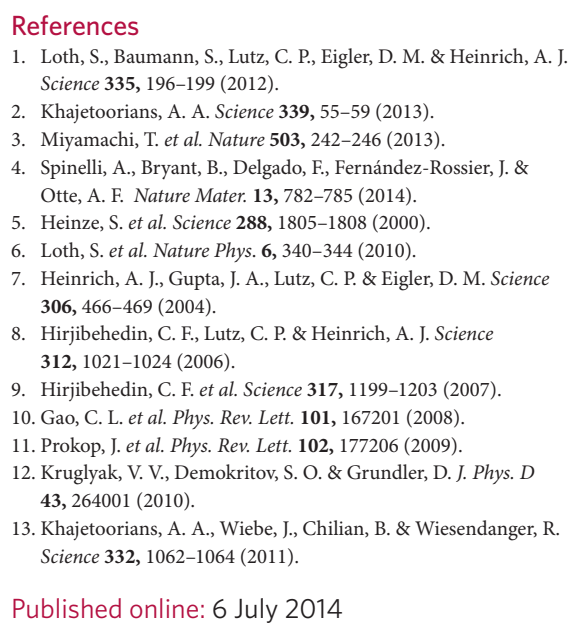

References

1. Loth, S., Baumann, S., Lutz, C. P., Eigler, D. M. \& Heinrich, A. J.

Spinelli, A., Bryant, B., Delgado, F., Fernández-Rossier, J. \&

e, A. F. Nature Mater. 13, 782-785 (2014).

.
306, 466-469 (2004)
2. Kruglyak, V. V., Demokritov, S. O. \& Grundler, D. J. Phys. D , 264001 (2010)

Published online: 6 July 2014

\title{
CUTTING-EDGE METALLURGY
}

There can be few more mythologized ancient materials technologies than sword-making. The common view - that ancient metalsmiths had an extraordinary empirical grasp of how to manipulate alloy microstructure to make the finestquality blades - contains a fair amount of truth. Perhaps the most remarkable example of this was discovered several years ago: the nearlegendary Damascus blades used by Islamic warriors, which were flexible yet strong and hard enough to cleave the armour of Crusaders, contained carbon nanotubes ${ }^{1}$. Formation of the nanotubes was apparently catalysed by impurities such as vanadium in the steel, and these nanostructures assisted the growth of cementite $\left(\mathrm{Fe}_{3} \mathrm{C}\right)$ fibres that thread through the unusually high-carbon steel known as wootz, making it hard without paying the price of brittleness.

Yet it seems that the skill of the swordsmith wasn't directed purely at making swords mechanically superior. Thiele et al. report ${ }^{2}$ that the practice called pattern welding, well established in swords from the second century AD to the early medieval period, was primarily used for decorative rather than mechanical purposes and, unless used with care, could even have compromised the quality of the blades.

Pattern welding involved the lamination and folding of two materials - high-phosphorus iron and low-phosphorus mild steel or iron - to produce a surface that could be polished and etched to striking decorative effect. After twisting and grinding, the metal surface could acquire striped, chevron and sinuous patterns that were highly prized. A letter to a Germanic tribe in the sixth century AD, complimenting them for the swords they gave to the Ostrogothic king Theodoric, conqueror of Italy, praised the interplay of shadows and colours in the blades, comparing the pattern to tiny snakes.

But was it all about appearance? Surely what mattered most to a warrior was that his sword could be relied on to slice, stab and maim without breaking? It seems not. Thiele et al. commissioned an internationally renowned swordsmith to make pattern-welded rods for them using traditional techniques and re-smelted medieval iron. In these samples the high-phosphorus component was iron and not, as some earlier studies have mistakenly assumed, steel.

They subjected the samples to mechanical tests that probed the stresses typically experienced by a sword: impact, bending and buckling. In no cases did the pattern-welded samples perform any better than hardened and tempered steel. This is not so surprising, given that phosphoric iron itself has rather poor toughness, no matter how it is

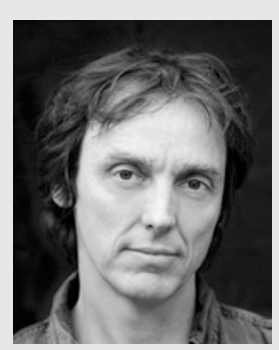

PHILIP BALL

laminated with other materials.

The prettiness of pattern welding didn't, however, have to compromise the sword's strength, as - at least in later examples - the patterned section was confined to panels in the central 'fuller' of the blade, while the cutting edge was steel. All the same, here's an example of how materials use may be determined as much by social as by technical and mechanical considerations. From the Early to the High Middle Ages, swords weren't just or even primarily for killing people with. For the Frankish warrior, the spear and axe were the main weapons; swords were largely symbols of power and status, carried by chieftains, jarls and princes but used only rarely. Judging by the modern reproductions, they looked almost too gorgeous to stain with blood.

References

1. Reibold, M. et al. Nature 444, 286 (2006).

2. Thiele, A., Hosek, J., Kucypera, P. \& Dévényi, L. Archaeometry http://dx.doi.org/10.1111/arcm.12114 (2014) 